\title{
Neutrino mass ordering determination through combined analysis with JUNO and KM3NeT/ORCA
}

\section{João Pedro Athayde Marcondes de André,,${ }^{a} *$ Nhan Chau,,${ }^{b}$ Marcos Dracos, ${ }^{a}$ Leonidas Kalousis, ${ }^{a}$ Antoine Kouchner ${ }^{b c}$ and Véronique Van Elewyck ${ }^{b c}$ on behalf of the KM3NeT Collaboration and members of the JUNO Collaboration}

(a complete list of authors can be found at the end of the proceedings)

${ }^{a} I P H C$, Université de Strasbourg, CNRS/IN2P3, F-67037 Strasbourg, France

${ }^{b}$ Université de Paris, CNRS, Astroparticule et Cosmologie, F-75013 Paris, France

${ }^{c}$ Institut Universitaire de France, 1 rue Descartes, F-75005 Paris, France

E-mail: jpandre@iphc.cnrs.fr, nchau@apc.in2p3.fr

The determination of neutrino mass ordering (NMO) is one of the prime goals of several neutrino experiments. KM3NeT/ORCA and JUNO are two next-generation neutrino oscillation experiments both aiming at addressing this question. ORCA determines the NMO by probing Earth matter effects on the oscillation of atmospheric neutrinos in the GeV energy range. JUNO, on the other hand, is sensitive to the NMO by investigating the interference effects of fast oscillations in the reactor electron antineutrino spectrum at medium baseline. This poster presents the potential of determining the NMO through a combined analysis of JUNO and ORCA data. When measuring the $\Delta m_{31}^{2}$ with a wrong ordering assumption, the best-fit values are different between the two experiments. This tension, together with good constraints on the $\Delta m_{31}^{2}$ measurement by both experiments, enhances the combined NMO sensitivity beyond the simple sum of their sensitivities. The analysis shows that $5 \sigma$ significance is reachable in less than 2 years of data taking with both experiements for true normal neutrino mass ordering assuming current global best-fit values of the oscillation parameters, while 6 years will be needed for any other parameter set.

$37^{\text {th }}$ International Cosmic Ray Conference (ICRC 2021)

July 12th - 23rd, 2021

Online - Berlin, Germany

\footnotetext{
${ }^{*}$ Presenter
} 


\section{Introduction}

The discovery of neutrino flavour oscillations at the beginning of the century $[1,2]$ demonstrated that neutrinos are massive particles. In total, 6 parameters are used to describe 3 flavour neutrino oscillations: 2 mass squared differences $\left(\Delta m_{21}^{2}\right.$, and $\left.\Delta m_{31}^{2}\right), 3$ mixing angles $\left(\theta_{12}, \theta_{13}\right.$, and $\left.\theta_{23}\right)$, and a CP-violating phase $\left(\delta_{\mathrm{CP}}\right)$. Since their discovery, significant progress has been made in the determination of these parameters [3]. However, at this moment, neither the existence of CPviolation in the neutrino sector, nor the order of the masses of the neutrinos, have been clearly determined. Indeed, as the sign of $\Delta m_{31}^{2}$ is still unknown there are two possibilities for the neutrino mass ordering (NMO). The first possibility, called "normal ordering", corresponds to the same ordering as the other fundamental particles from the Standard Model of Particle Physics, that is, $m_{1}<m_{2}<m_{3}$. The second possibility, called "inverted ordering", corresponds to taking $m_{3}<m_{1}<m_{2}$ which effectively means that the $v_{e}$ would not be the lightest neutrino.

The measurement of the NMO is on the agenda of several existing and planned neutrino experiments. JUNO [4, 5] and KM3NeT/ORCA [6] are two next-generation neutrino detectors that plan to address this question. Both detectors are already under construction and both expect to be fully functional and taking data by 2025. ORCA, the low-energy branch of the KM3NeT neutrino telescope, will determine the NMO by probing Earth matter effects on the oscillations of atmospheric neutrinos. JUNO is a medium-baseline reactor neutrino experiment which is sensitive to the NMO through the precise measurement of the fast oscillations in the spectrum of the reactor electron anti-neutrino spectra, where matter effects play only a small role.

Thanks to the complementarity in their approaches to measuring the NMO, combining JUNO and ORCA yields an improvement in sensitivity beyond the one from simply adding in quadrature their sensitivities. This happens due to the tension that appears between the best-fit values of $\Delta m_{31}^{2}$ from either experiment under the wrong NMO assumption. This effect had already been pointed out previously from the combination of reactor and accelerator $[7,8]$ or atmospheric $[9,10]$ neutrinos. A study performed using the latest estimated performance of both JUNO and ORCA is presented in this proceedings.

\section{Jiangmen Underground Neutrino Observatory (JUNO)}

The Jiangmen Underground Neutrino Observatory $[4,5]$ is a multipurpose experiment being built in the south of China. One of its goals is the determination of the NMO via the precise measurement of electron anti-neutrino oscillations from the Yangjiang and the Taishan Nuclear Power Plants (NPP) located $53 \mathrm{~km}$ away from the detector.

The JUNO detector is divided in 3 parts: the Central Detector, the Water Cherenkov Detector and the Top Tracker. The last two parts compose the Veto System of JUNO, while the first part is the main neutrino target. The Central Detector is composed of $20 \mathrm{kton}$ of liquid scintillator placed in a $35.4 \mathrm{~m}$ diameter acrylic sphere. Around this acrylic sphere, about 1800020 " and about 26000 3" photomultiplier tubes (PMT) monitor the liquid scintillator in order to detect neutrino interactions occurring inside it. Of particular interest are the Inverse Beta Decay (IBD) interactions produced by electron anti-neutrinos from the NPPs. The IBD interactions are detected in the JUNO Central Detector via the prompt detection of the scintillation light of the positron produced in the 


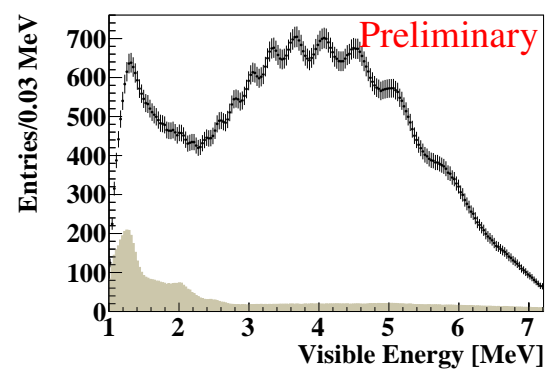

Figure 1: Expected event distribution for 6 years of data with the JUNO with 6 Yangiiang and 2 Taishan reactor cores as a function of the visible energy of the prompt signal. The current world best-fit [13] oscillation parameters for normal ordering are assumed. The shaded region corresponds to the non-reactor neutrino background events.

interaction and its subsequent annihilation, along with the delayed detection of a $2.2 \mathrm{MeV}$ gammaray produced in the neutron capture on hydrogen and subsequent de-excitation of the deuteron. This design of the JUNO Central Detector is crucial to reach the energy resolution of $3 \% / \sqrt{E / \mathrm{MeV}}$ required for the determination of the NMO at $3 \sigma$.

As discussed previously, the Yangjiang and Taishan NPPs are the two main NPPs contributing to the reactor neutrino flux observed by JUNO. In addition to those NPPs, JUNO observes also neutrinos from the Daya-Bay and Huizhou NPPs which are located more than $200 \mathrm{~km}$ away from the detector. However, given this longer baseline, the neutrinos from these NPPs will effectively be an intrinsic background to the NMO analysis. All reactor cores from the Daya-Bay and Huizhou NPPs are considered to be operational in this analysis.

At the time of the writing of Ref. [4], it was expected that Yangjiang would host 6 reactor cores and Taishan would host 4 reactor cores. While most of these reactor cores have already been built, the plans for adding the last 2 of Taishan cores is currently uncertain [5]. To be conservative, the total number of cores providing signal $\bar{v}_{e}$ used in this study is then reduced to 8 cores, excluding the 2 not yet constructed Taishan reactors, from the initial 10 cores in Ref. [4].

Given the risk of possible unknown substructures in the reactor neutrino spectra [11], the JUNO project was augmented to include the JUNO-TAO detector [12]. This detector will be installed at a distance of $30 \mathrm{~m}$ from one of the Taishan's reactors to determine the reactor electron anti-neutrino spectrum with a better energy resolution than JUNO. Thanks to this reference spectrum, the impact of these possible unknown substructures is reduced and will not be considered in this study.

The JUNO analysis used in this study follows the same modeling of the experiment explained in Ref. [4]. The notable departures from that description are in the number of Taishan NPP available number of cores, as described above, and in the oscillation parameters, whose values are used from a recent global fit result presented in Ref. [13]. The expected event distribution as a function of the visible energy for JUNO is shown in Fig. 1.

\section{Oscillation Research with Cosmics in the Abyss (ORCA)}

The KM3NeT Collaboration is currently building a set of next-generation water Cherenkov telescopes in the depths of the Mediterranean Sea [6]. Two separate arrays of PMTs will be 

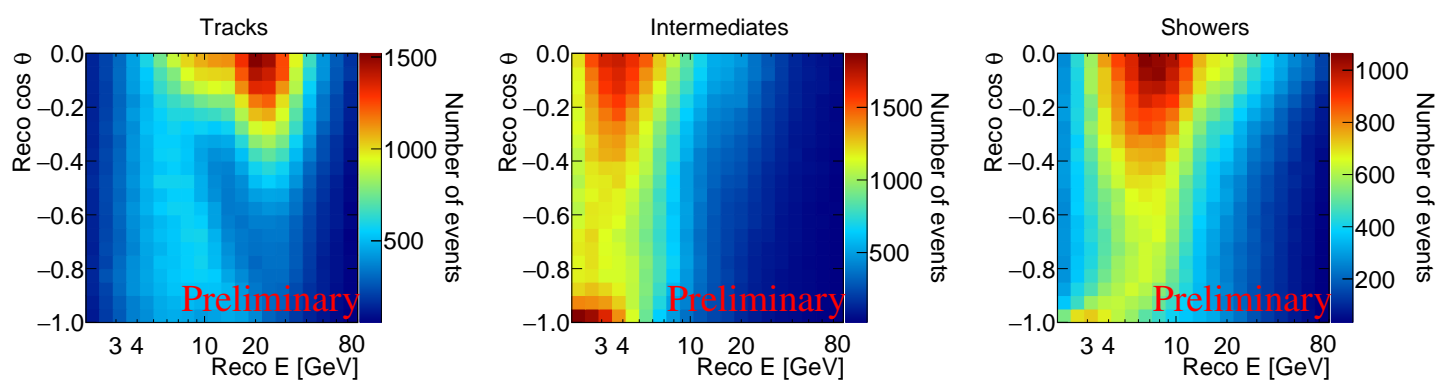

Figure 2: Expected event distribution for ORCA in 3 PID classes for 6 years of exposure and true NO assumption, with the oscillation parameter values from Ref. [13].

deployed: ARCA and ORCA. ARCA is a gigaton-scale detector whose purpose is mainly to search for astrophysical neutrinos in the TeV-PeV energy range. ORCA, which is the subject of this study, is a denser and smaller array (Mton-scale) optimized for oscillation physics with atmospheric neutrinos at energies greater than $1 \mathrm{GeV}$.

The ORCA detector will contain 115 detection units anchored to the seabed. Each detection unit is composed of a $200 \mathrm{~m}$ long slender vertical line supporting 18 digital optical modules, with a vertical spacing of around $9 \mathrm{~m}$. Each digital optical module is a glass sphere containing 31 PMTs and related electronics. The detection units are disposed in a cylindrical shape with an average radius of $115 \mathrm{~m}$ and an average distance between the units of $20 \mathrm{~m}$. In total, the instrumented mass of ORCA is of about 7.0 Mton.

ORCA will detect the Cherenkov light from charged particles produced by the interaction of atmospheric neutrino within the detector and its surroundings. These interactions, in the case of ORCA, will typically be composed of charged current (CC) and neutral current (NC) processes off nucleons in water molecules. The pattern and timing of the digitalized PMT output are used to identify neutrino events and reconstruct their energy and angular direction. The topology of these events is also used to separate the interactions in two different classes: tracks (when an individual, energetic muon can be identified in the event) and showers (in all other cases). Track-like events are expected in the case of CC interactions from $v_{\mu} / \bar{v}_{\mu}$ and from some $v_{\tau} / \bar{v}_{\tau}$. Shower-like events are associated to NC interactions and for CC interactions from $v_{e} / \bar{v}_{e}$ and the remainder of those from $v_{\tau} / \bar{v}_{\tau}$. Effectively, a PID variable is built to separate these signatures and three classifications are created: track-like, intermediate, and shower-like. The track-like and shower-like classifications correspond to the two signatures described above, while the intermediate classification includes events not clearly in either sample.

The ORCA detector is modeled for this study using the official KM3NeT collaboration tools. A full Monte Carlo simulation [14, 15] of the detector response to atmospheric neutrinos was used to generate interactions in the $1-100 \mathrm{GeV}$ energy range. The Monte Carlo sample, event selection, and reconstruction used in this study are identical to those used and described extensively in Ref. [16]. The expected event distributions, for each PID classification, are shown in Fig. 2 as a function of their reconstructed energy and direction.

The systematic errors considered for ORCA are of particular importance for this analysis. In the baseline scenario, the same systematic errors as in Ref. [16] are used. In addition to the baseline scenario, an optimistic scenario is also considered based on the study performed in Ref. [10] for 
Table 1: Baseline and optimistic scenarios for the treatment of systematics considered in the ORCA analysis.

\begin{tabular}{c|c|c} 
Parameter & Baseline scenario & Optimistic scenario \\
\hline PID-class norm. factors & free & $\times$ \\
Effective area scale & $\times$ & $10 \%$ prior \\
Detector energy scale & $5 \%$ prior & $\times$ \\
Flux energy scale & $\times$ & $10 \%$ prior \\
Flux $v_{e} / \bar{v}_{e}$ skew & \multicolumn{2}{|c}{$7 \%$ prior } \\
Flux $v_{\mu} / \bar{v}_{\mu}$ skew & \multicolumn{2}{|c}{$5 \%$ prior } \\
Flux $v_{e} / \bar{v}_{\mu}$ skew & \multicolumn{2}{|c}{$2 \%$ prior } \\
Flux spectral index & \multicolumn{2}{|c}{ free } \\
NC normalization & \multicolumn{2}{|c}{$10 \%$ prior } \\
\hline
\end{tabular}

the PINGU detector. Both scenarios are shown in Tab. 1. In the optimistic scenario the energy scale uncertainty is introduced as an uncertainty in the neutrino flux rather than as an uncertainty at the detection level. The normalization uncertainty is also treated differently in each scenario: in the optimistic scenario only a global normalization is introduced, while in the baseline scenario separate normalization factors are introduced for each PID class. It is worth noting that the baseline scenario is believed to best describe the real uncertainties in the ORCA detector. When it is not specified explicitly, the baseline scenario is used in the results.

\section{Combined analysis}

As described in Secs. 2 and 3, each detector works in very different conditions, with a neutrino flux from different sources and with different energy ranges. Given these large differences, the only common parameters to both experiments are the neutrino oscillation parameters.

It is also worth noting that not all neutrino oscillation parameters affect both experiments. On one hand, the $\bar{v}_{e} \rightarrow \bar{v}_{e}$ oscillations observed by JUNO do not depend on either $\theta_{23}$ or $\delta_{\mathrm{CP}}$, however they need to be considered by ORCA. In particular, the true value $\theta_{23}$ has a strong impact in the ORCA results and in the analysis performed by ORCA it is important to fit the $\theta_{23}$ parameter twice, each time starting from a different octant, to guarantee the fit finds the global $\chi^{2}$ minimum. On the other hand, the atmospheric neutrino oscillations observed by ORCA are at a much smaller $L / E$ than those required for the development of the oscillation with frequency given by $\Delta m_{21}^{2}$. This means that ORCA has negligible sensitivity to $\Delta m_{21}^{2}$ and $\theta_{12}$, which are also parameters that JUNO will be able to measure to a sub-percent precision. Finally, both experiments are sensitive to the two remaining parameters, $\Delta m_{31}^{2}$ and $\theta_{13}$. These two parameters will, therefore, have to be explicitly included in the combined analysis, while the other four parameters are treated separately in the analyses of each experiment.

For the combined analysis, a grid scan on $\Delta m_{31}^{2}$ and $\theta_{13}$ is performed. At each node of the grid, an Asimov data set [17] for each experiment is used to compute each experiment's $\chi^{2}$ value by profiling over all systematic parameters and for the oscillation parameters that are not globally relevant. These $\chi^{2}$ values, at each node of the grid, are then added to create a combined $\chi^{2}$, as shown in Eq. (1). A prior term is added to this $\chi^{2}$ to account for the current precision of $\theta_{13}$ from 

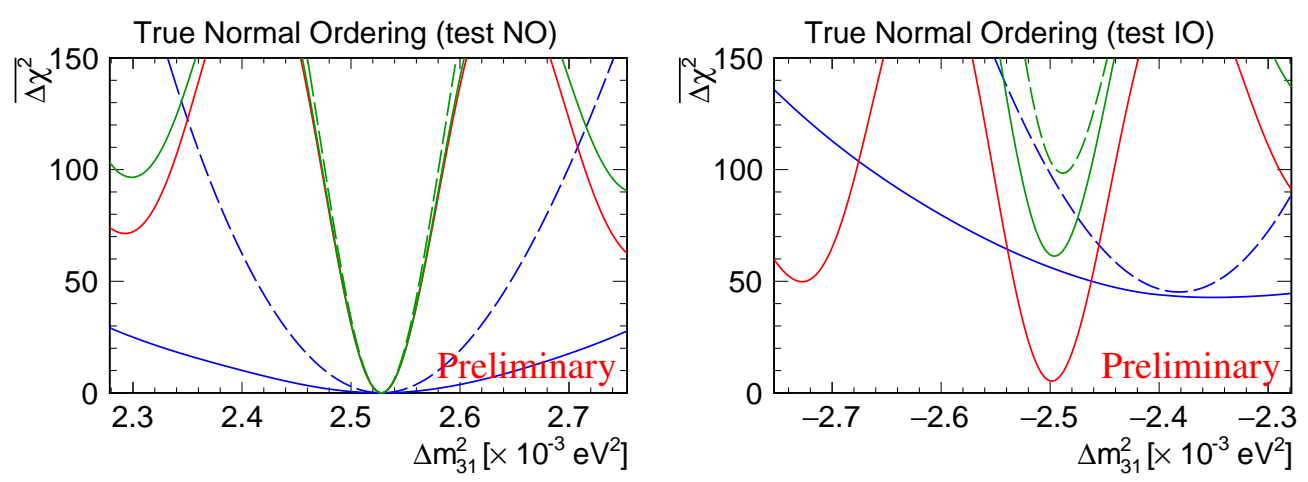

Figure 3: $\overline{\Delta \chi^{2}}$ profile for only JUNO (red), only ORCA (blue), and the combination of JUNO and ORCA (green) as a function of test values of $\Delta m_{31}^{2}$ for 6 years of data taking assuming baseline (solid) or optimistic (dashed) systematics.

reactor neutrino experiments [18-20], which is better than the expected precision from either JUNO or ORCA. The best-fit value and uncertainty from Ref. [13] are used in this prior.

$$
\chi^{2}\left(\Delta m_{31}^{2}, \theta_{13}\right)=\chi_{\mathrm{JUNO}}^{2}\left(\Delta m_{31}^{2}, \theta_{13}\right)+\chi_{\mathrm{ORCA}}^{2}\left(\Delta m_{31}^{2}, \theta_{13}\right)+\frac{\left(\sin ^{2} \theta_{13}-\sin ^{2} \theta_{13}^{\mathrm{GF}}\right)^{2}}{\sigma_{\sin ^{2} \theta_{13}^{\mathrm{GF}}}^{2}}
$$

The resulting $\chi^{2}$ map is then used to estimate the combined analysis sensitivity to the NMO by calculating the $\overline{\Delta \chi^{2}}$ as the difference between the minimum $\chi^{2}$ values obtained from the wrong and true orderings. In Fig. 3 is shown one example of this $\overline{\Delta \chi^{2}}$ profile obtained for the best-fit value for normal ordering given in Ref. [13]. In this figure are also shown the results obtained by the stand-alone JUNO and ORCA analyses for comparison.

From Fig. 3, the reason why the combination of JUNO and ORCA data significantly boost the NMO sensitivity becomes clear: while both experiments obtain the same best-fit value for $\Delta m_{31}^{2}$ under the true ordering, in the wrong ordering a tension appears between these best-fit values. The requirement that there is only one best-fit $\Delta m_{31}^{2}$ in the combined analysis therefore helps to further reject the wrong ordering hypothesis and increases its sensitivity. It is also worth noting, from Fig. 3, that the systematic errors used in ORCA have a large impact in the final sensitivity as they strongly impact ORCA's expected precision on $\Delta m_{31}^{2}$, even if they have only a small impact on the stand-alone sensitivity of ORCA to the NMO.

\section{Results and Conclusion}

The sensitivity of this combined analysis as a function of time is shown in Fig. 4, assuming the best-fit values from Ref. [13] as the true parameters for each ordering. For normal ordering, a $5 \sigma$ sensitivity is reached with 2 years of data with both detectors in their final configurations assuming the baseline scenario systematic errors for ORCA, which is a full year earlier than what ORCA would obtain by itself. Assuming the optimistic scenario would further reduce by almost a year the time to reach $5 \sigma$. In the inverted ordering case, a $5 \sigma$ sensitivity is reached after 6 years of 

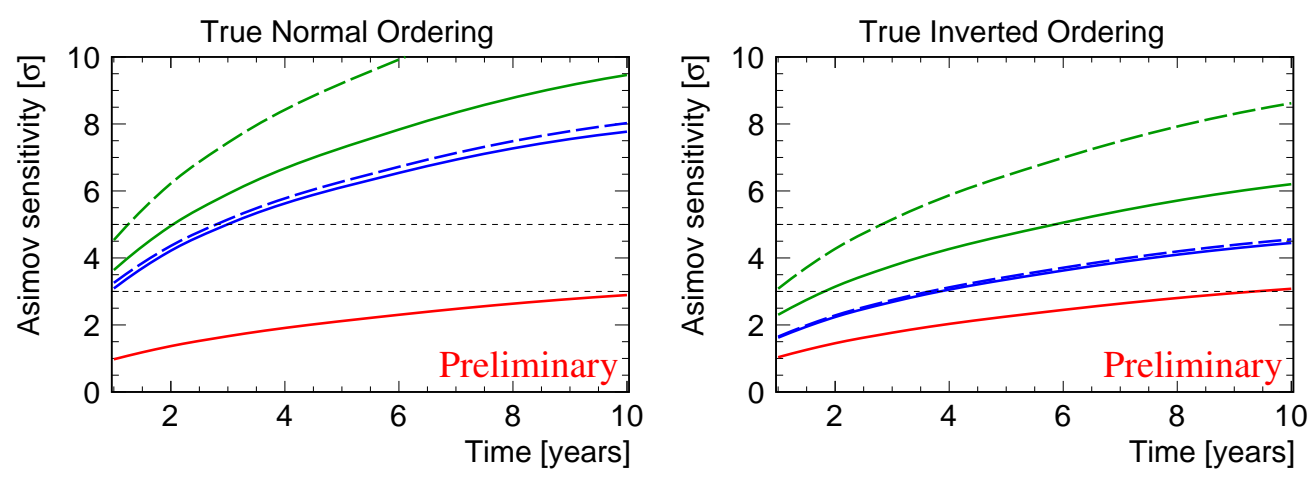

Figure 4: NMO sensitivity as a function of time for only JUNO (red), only ORCA (blue), and the combination of JUNO and ORCA (green), assuming baseline (solid) or optimistic (dashed) systematics.
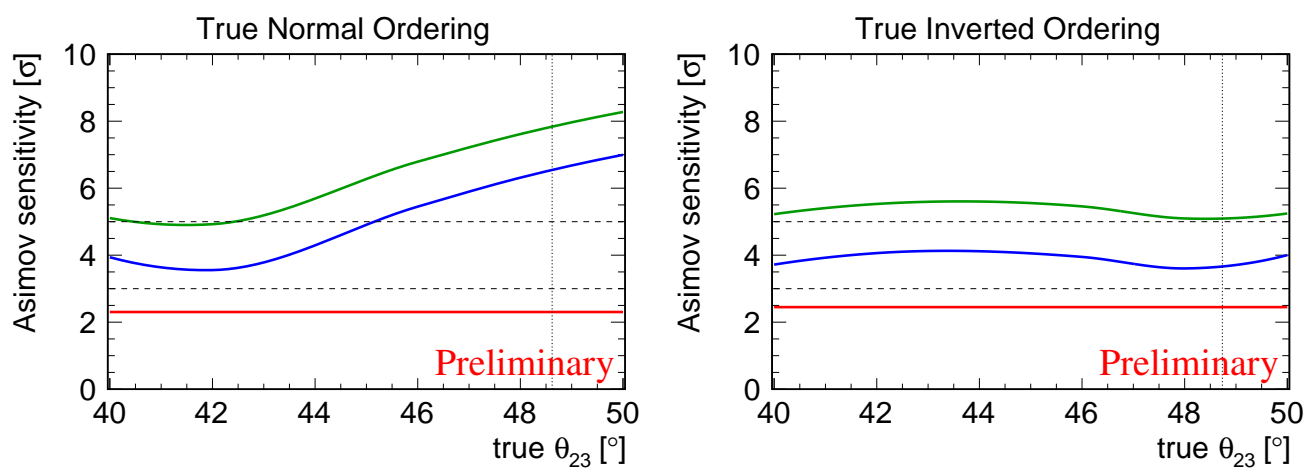

Figure 5: NMO sensitivity as a function of the true $\theta_{23}$ value for 6 years of data taking for only JUNO (red), only ORCA (blue), and the combination of JUNO and ORCA (green). The vertical lines indicate the global best-fit values used in this analysis (from Ref. [13]).

data from both experiments, which is a large improvement from the more than 10 years required by ORCA alone.

As mentioned previously, the ORCA sensitivity depends strongly on the true value of $\theta_{23}$. Fig. 5 shows how the sensitivity, with 6 years of data from ORCA and JUNO, depends on the true value of $\theta_{23}$. From this figure, the current best-fit value for normal ordering corresponds to one of the values where ORCA is the most sensitive, while the current best-fit value for inverted ordering corresponds to one of the cases where the sensitivity is the smallest. It is important to note that for all true values of $\theta_{23}$, in both orderings, the NMO is determined at $5 \sigma$ by the combined analysis of 6 years of data from JUNO and ORCA.

Finally, as was highlighted in Sec. 2, the energy resolution is a critical parameter for the success of the JUNO determination of the NMO. While it is fully expected that JUNO will reach the desired precision [21], in the combined analysis the impact from the JUNO energy resolution in the result is significantly reduced. This is due to the fact that the values of wrong ordering best-fits from JUNO and ORCA do not significantly change and therefore the tension is only slightly reduced from a worsening of the precision of JUNO's $\Delta m_{31}^{2}$ determination. 

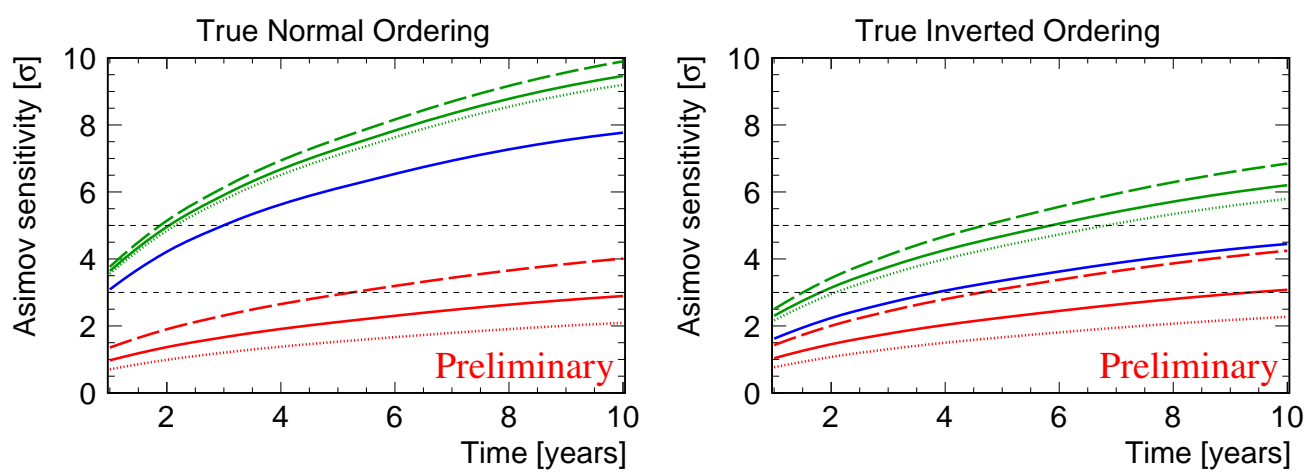

Figure 6: NMO sensitivity as a function of time for only JUNO (red), only ORCA (blue), and the combination of JUNO and ORCA (green), considering a better (dashed) and worse (dotted) energy resolution for JUNO than the nominal one (solid) by $\pm 0.5 \% / \sqrt{E / \mathrm{MeV}}$.

\section{Acknowledgements}

The authors acknowledge the financial support of the Centre National de la Recherche Scientifique (CNRS), the Agence Nationale de la Recherche (contract ANR-15-CE31-0020) and the LabEx UnivEarthS (ANR-10-LABX-0023 and ANR-18-IDEX-0001).

\section{References}

[1] Y. Fukuda et al. (Super-Kamiokande Collaboration), Phys. Rev. Lett., 81:1562-1567, 1998.

[2] Q. R. Ahmad et al. (SNO Collaboration), Phys. Rev. Lett., 89:011301, 2002.

[3] P. A. Zyla et al. (Particle Data Group Collaboration), PTEP, 2020(8):083C01, 2020.

[4] F. An et al. (JUNO Collaboration), J. Phys. G, 43(3):030401, 2016.

[5] A. Abusleme et al. (JUNO Collaboration). arXiv:2104.02565, 2021.

[6] S. Adrian-Martinez et al. (KM3Net Collaboration), J. Phys. G, 43(8):084001, 2016.

[7] H. Nunokawa et al., Phys. Rev. D, 72:013009, 2005.

[8] A. de Gouvea et al., Phys. Rev. D, 71:113009, 2005.

[9] M. Blennow et al., JHEP, 09:089, 2013.

[10] M. Aartsen et al. (JUNO Collaboration members and IceCube Gen2 Collaboration), Phys. Rev. D, 101(3):032006, 2020.

[11] D. A. Dwyer et al., Phys. Rev. Lett., 114(1):012502, 2015.

[12] A. Abusleme et al. (JUNO Collaboration). arXiv:2005.08745, 2020.

[13] I. Esteban et al., JHEP, 01:106, 2019.

[14] S. Aiello et al. (KM3NeT Collaboration), Comput. Phys. Commun., 256:107477, 2020.

[15] A. Tsirigotis et al., Nucl. Instrum. Meth. A, 626-627:S185-S187, 2011.

[16] S. Aiello et al. (KM3NeT Collaboration). arXiv:2103.09885, 2021. Submitted to EPJ. C.

[17] G. Cowan et al., Eur. Phys. J. C, 71:1554, 2011. [Erratum: Eur.Phys.J.C 73, 2501 (2013)].

[18] D. Adey et al. (Daya Bay Collaboration), Phys. Rev. Lett., 121(24):241805, 2018.

[19] G. Bak et al. (RENO Collaboration), Phys. Rev. Lett., 121(20):201801, 2018.

[20] H. de Kerret et al. (Double Chooz Collaboration), Nature Phys., 16(5):558-564, 2020.

[21] A. Abusleme et al. (JUNO Collaboration), JHEP, 03:004, 2021. 


\section{Full Authors List:}

\section{KM3NeT Collaboration}

M. Ageron ${ }^{1}$, S. Aiello ${ }^{2}$, A. Albert ${ }^{3,55}$, M. Alshamsi ${ }^{4}$, S. Alves Garre ${ }^{5}$,Z. Aly ${ }^{1}$, A. Ambrosone ${ }^{6,7}$, F. Ameli ${ }^{8}$, M. Andre ${ }^{9}$, G. Androulakis ${ }^{10}$, M. Anghinolfi ${ }^{11}$, M. Anguita ${ }^{12}$, G. Anton ${ }^{13}$, M. Ardid $^{14}$, S. Ardid $^{14}$, W. Assal ${ }^{1}$, J. Aublin ${ }^{4}$, C. Bagatelas ${ }^{10}$, B. Baret ${ }^{4}$, S. Basegmez du Pree ${ }^{15}$, M. Bendahman ${ }^{4,16}$, F. Benfenati ${ }^{17,18}$, E. Berbee ${ }^{15}$, A. M. van den Berg ${ }^{19}$, V. Bertin ${ }^{1}$, S. Beurthey ${ }^{1}$, V. van Beveren ${ }^{15}$, S. Biagi ${ }^{20}$, M. Billault ${ }^{1}$, M. Bissinger ${ }^{13}$, M. Boettcher ${ }^{21}$, M. Bou Cabo ${ }^{22}$, J. Boumaaza ${ }^{16}$, M. Bouta ${ }^{23}$, C. Boutonnet ${ }^{4}$, G. Bouvet ${ }^{24}$, M. Bouwhuis ${ }^{15}$, C. Bozza ${ }^{25}$, H.Brânzaş ${ }^{26}$, R. Bruijn ${ }^{15,27}$, J. Brunner ${ }^{1}$, R. Bruno ${ }^{2}$, E. Buis ${ }^{28}$, R. Buompane ${ }^{6,29}$, J. Busto ${ }^{1}$, B. Caiffí ${ }^{11}$, L. Caillat ${ }^{1}$, D. Calvo ${ }^{5}$, S. Campion ${ }^{30,8}$, A. Capone ${ }^{30,8}$, H. Carduner ${ }^{24}$, V. Carretero ${ }^{5}$, P. Castaldi ${ }^{17,31}$, S. Celli ${ }^{30,8}$, R. Cereseto ${ }^{11}$, M. Chabab ${ }^{32}$, C. Champion ${ }^{4}$, N. Chau ${ }^{4}$, A. Chen ${ }^{33}$, S. Cherubini ${ }^{20,34}$, V. Chiarella ${ }^{35}$, T. Chiarusi ${ }^{17}$, M. Circella ${ }^{36}$, R. Cocimano ${ }^{20}$, J. A. B. Coelho ${ }^{4}$, A. Coleiro ${ }^{4}$, M. Colomer Molla ${ }^{4,5}$, S. Colonges ${ }^{4}$, R. Coniglione ${ }^{20}$, A. Cosquer ${ }^{1}$, P. Coyle ${ }^{1}$, M. Cresta ${ }^{11}$, A. Creusot ${ }^{4}$, A. $\mathrm{Cruz}^{37}$, G. Cuttone ${ }^{20}$, A. D’Amico ${ }^{15}$, R. Dallier ${ }^{24}$, B. De Martino ${ }^{1}$, M. De Palma ${ }^{36,38}$, I. Di Palma ${ }^{30,8}$, A. F. Díaz ${ }^{12}$, D. Diego$\operatorname{Tortosa}^{14}$, C. Distefano ${ }^{20}$, A. Domi ${ }^{15,27}$, C. Donzaud ${ }^{4}$, D. Dornic ${ }^{1}$, M. Dörr ${ }^{39}$, D. Drouhin ${ }^{3,55}$, T. Eberl ${ }^{13}$, A. Eddyamoui ${ }^{16}$, T. van Eeden ${ }^{15}$, D. van Eijk ${ }^{15}$, I. El Bojaddaini ${ }^{23}$, H. Eljarrari ${ }^{16}$, D. Elsaesser ${ }^{39}$, A. Enzenhöfer ${ }^{1}$, V. Espinosa ${ }^{14}$, P. Fermani ${ }^{30,8}$, G. Ferrara ${ }^{20,34}$, M. D. Filipović ${ }^{40}$, F. Filippini ${ }^{17,18}$, J. Fransen ${ }^{15}$, L. A. Fusco ${ }^{1}$, D. Gajanana ${ }^{15}$, T. Gal ${ }^{13}$, J. García Méndez ${ }^{14}$, A. Garcia Soto $^{5}$, E. Garçon ${ }^{1}$, F. Garufi ${ }^{6,7}$, C. Gatius ${ }^{15}$, N. Geißelbrecht ${ }^{13}$, L. Gialanella ${ }^{6,29}$, E. Giorgio ${ }^{20}$, S. R. Gozzini ${ }^{5}$, R. Gracia ${ }^{15}$, K. Graf ${ }^{13}$, G. Grella ${ }^{41}$, D. Guderian ${ }^{56}$, C. Guidi ${ }^{11,42}$, B. Guillon ${ }^{43}$, M. Gutiérrez ${ }^{44}$, J. Haefner ${ }^{13}$, S. Hallmann ${ }^{13}$, H. Hamdaoui ${ }^{16}$, H. van Haren ${ }^{45}$, A. Heijboer ${ }^{15}$, A. Hekalo ${ }^{39}$, L. Hennig ${ }^{13}$, S. Henry ${ }^{1}$, J. J. Hernández-Rey ${ }^{5}$, J. Hofestädt ${ }^{13}$, F. Huang ${ }^{1}$, W. Idrissi Ibnsalih ${ }^{6,29}$, A. Ilioni ${ }^{4}$, G. Illuminati ${ }^{17,18,4}$, C. W. James ${ }^{37}$, D. Janezashvili ${ }^{46}$, P. Jansweijer ${ }^{15}$, M. de Jong ${ }^{15,47}$, P. de Jong ${ }^{15,27}$, B. J. Jung ${ }^{15}$, M. Kadler ${ }^{39}$, P. Kalaczyński ${ }^{48}$, O. Kalekin ${ }^{13}$, U. F. Katz ${ }^{13}$, F. Kayzel ${ }^{15}$, P. Keller ${ }^{1}$, N. R. Khan Chowdhury ${ }^{5}$, G. Kistauri ${ }^{46}$, F. van der Knaap ${ }^{28}$, P. Kooijman ${ }^{27,57}$, A. Kouchner ${ }^{4,49}$, M. Kreter ${ }^{21}$, V. Kulikovskiy ${ }^{11}$, M. Labalme ${ }^{43}$, P. Lagier ${ }^{1}$, R. Lahmann ${ }^{13}$, P. Lamare ${ }^{1}$, M. Lamoureux $^{\ddagger 4}$, G. Larosa ${ }^{20}$, C. Lastoria ${ }^{1}$, J. Laurence ${ }^{1}$, A. Lazo $^{5}$, R. Le Breton ${ }^{4}$, E. Le Guirriec ${ }^{1}$, S. Le Stum ${ }^{1}$, G. Lehaut ${ }^{43}$, O. Leonardi ${ }^{20}$, F. Leone ${ }^{20,34}$, E. Leonora ${ }^{2}$, C. Lerouvillois ${ }^{1}$, J. Lesrel ${ }^{4}$, N. Lessing ${ }^{13}$, G. Levi ${ }^{17,18}$, M. Lincetto ${ }^{1}$, M. Lindsey Clark ${ }^{4}$, T. Lipreau ${ }^{24}$, C. LLorens Alvarez ${ }^{14}$, A. Lonardo ${ }^{8}$, F. Longhitano ${ }^{2}$, D. Lopez-Coto ${ }^{44}$, N. Lumb ${ }^{1}$, L. Maderer ${ }^{4}$, J. Majumdar ${ }^{15}$, J. Mańczak ${ }^{5}$, A. Margiotta ${ }^{17,18}$, A. Marinelli ${ }^{6}$, A. Marini ${ }^{1}$, C. Markou $^{10}$, L. Martin ${ }^{24}$, J. A. Martínez-Mora ${ }^{14}$, A. Martini ${ }^{35}$, F. Marzaioli ${ }^{6,29}$, S. Mastroianni ${ }^{6}$, K. W. Melis ${ }^{15}$, G. Miele ${ }^{6,7}$, P. Migliozzi ${ }^{6}$, E. Migneco ${ }^{20}$, P. Mijakowski ${ }^{48}$, L. S. Miranda ${ }^{50}$, C. M. Mollo ${ }^{6}$, M. Mongelli ${ }^{36}$, A. Moussa ${ }^{23}$, R. Muller ${ }^{15}$, P. Musico ${ }^{11}$, M. Musumeci ${ }^{20}$, L. Nauta ${ }^{15}$, S. Navas ${ }^{44}$, C. A. Nicolau ${ }^{8}$, B. Nkosi ${ }^{33}$, B. Ó Fearraigh ${ }^{15,27}$, M. O’Sullivan ${ }^{37}$, A. Orlando ${ }^{20}$, G. Ottonello ${ }^{11}$, S. Ottonello ${ }^{11}$, J. Palacios González ${ }^{5}$, G. Papalashvili ${ }^{46}$, R. Papaleo ${ }^{20}$, C. Pastore ${ }^{36}$, A. M. Păun ${ }^{26}$, G.E. Păvălaşş, G. Pellegrinini ${ }^{17}$, C. Pellegrino ${ }^{18,58}$, M. Perrin-Terrin ${ }^{1}$, V. Pestel ${ }^{15}$, P. Piattelli ${ }^{20}$, C. Pieterse ${ }^{5}$, O. Pisanti ${ }^{6,7}$, C. Poirè ${ }^{14}$, V. Popa $^{26}$, T. Pradier $^{3}$, F. Pratolongo ${ }^{11}$, I. Probst ${ }^{13}$, G. Pühlhofer ${ }^{51}$, S. Pulvirenti ${ }^{20}$, G. Quéméner ${ }^{43}$, N. Randazzo ${ }^{2}$, A. Rapicavoli ${ }^{34}$, S. Razzaque $^{50}$, D. Real ${ }^{5}$, S. Reck ${ }^{13}$, G. Riccobene ${ }^{20}$, L. Rigalleau ${ }^{24}$, A. Romanov ${ }^{11,42}$, A. Rovelli ${ }^{20}$, J. Royon ${ }^{1}$, F. Salesa Greus ${ }^{5}$, D. F. E. Samtleben ${ }^{15,47}$, A. Sánchez Losa ${ }^{36,5}$, M. Sanguineti ${ }^{11,42}$, A. Santangelo ${ }^{51}$, D. Santonocito ${ }^{20}$, P. Sapienza ${ }^{20}$, J. Schmelling ${ }^{15}$, J. Schnabel ${ }^{13}$, M. F. Schneider ${ }^{13}$, J. Schumann ${ }^{13}$, H. M. Schutte ${ }^{21}$, J. Seneca ${ }^{15}$, I. Sgura ${ }^{36}$, R. Shanidze ${ }^{46}$, A. Sharma ${ }^{52}$, A. Sinopoulou ${ }^{10}$, B. Spisso ${ }^{41,6}$, M. Spurio ${ }^{17,18}$, D. Stavropoulos ${ }^{10}$, J. Steijger ${ }^{15}$, S. M. Stellacci ${ }^{41,6}$, M. Taiuti ${ }^{11,42}$, F. Tatone ${ }^{36}$, Y. Tayalati ${ }^{16}$, E. Tenllado ${ }^{44}$, D. Tézier ${ }^{1}$, T. Thakore ${ }^{5}$, S. Theraube ${ }^{1}$, H. Thiersen $^{21}$, P. Timmer $^{15}$, S. Tingay ${ }^{37}$, S. Tsagkli $^{10}$, V. Tsourapis ${ }^{10}$, E. Tzamariudaki ${ }^{10}$, D. Tzanetatos ${ }^{10}$, C. Valieri ${ }^{17}$, V. Van Elewyck ${ }^{4,49}$, G. Vasileiadis ${ }^{53}$, F. Versari ${ }^{17,18}$, S. Viola $^{20}$, D. Vivolo ${ }^{6,29}$, G. de Wasseige ${ }^{4}$, J. Wilms ${ }^{54}$, R. Wojaczyński ${ }^{48}$, E. de Wolf ${ }^{15,27}$, T. Yousfi ${ }^{23}$, S. Zavatarelli ${ }^{11}$, A. Zegarelli ${ }^{30,8}$, D. Zito ${ }^{20}$, J. D. Zornoza ${ }^{5}$, J. Zúñiga ${ }^{5}$, N. Zywucka ${ }^{21}$.

${ }^{1}$ Aix Marseille Univ, CNRS/IN2P3, CPPM, Marseille, France.

${ }^{2}$ INFN, Sezione di Catania, Via Santa Sofia 64, Catania, 95123 Italy.

${ }^{3}$ Université de Strasbourg, CNRS, IPHC UMR 7178, F-67000 Strasbourg, France.

${ }^{4}$ Université de Paris, CNRS, Astroparticule et Cosmologie, F-75013 Paris, France.

${ }^{5}$ IFIC - Instituto de Física Corpuscular (CSIC - Universitat de València), c/Catedrático José Beltrán, 2, 46980 Paterna, Valencia, Spain. ${ }^{6}$ INFN, Sezione di Napoli, Complesso Universitario di Monte S. Angelo, Via Cintia ed. G, Napoli, 80126 Italy.

${ }^{7}$ Università di Napoli “Federico II", Dip. Scienze Fisiche "E. Pancini”, Complesso Universitario di Monte S. Angelo, Via Cintia ed. G, Napoli, 80126 Italy.

${ }^{8}$ INFN, Sezione di Roma, Piazzale Aldo Moro 2, Roma, 00185 Italy.

${ }^{9}$ Universitat Politècnica de Catalunya, Laboratori d'Aplicacions Bioacústiques, Centre Tecnològic de Vilanova i la Geltrú, Avda. Rambla Exposició, s/n, Vilanova i la Geltrú, 08800 Spain.

${ }^{10}$ NCSR Demokritos, Institute of Nuclear and Particle Physics, Ag. Paraskevi Attikis, Athens, 15310 Greece.

${ }^{11}$ INFN, Sezione di Genova, Via Dodecaneso 33, Genova, 16146 Italy.

${ }^{12}$ University of Granada, Dept. of Computer Architecture and Technology/CITIC, 18071 Granada, Spain.

${ }^{13}$ Friedrich-Alexander-Universität Erlangen-Nürnberg, Erlangen Centre for Astroparticle Physics, Erwin-Rommel-Straße 1, 91058 Erlangen, Germany.

${ }^{14}$ Universitat Politècnica de València, Instituto de Investigación para la Gestión Integrada de las Zonas Costeras, C/ Paranimf, 1, Gandia, 46730 Spain.

${ }^{15}$ Nikhef, National Institute for Subatomic Physics, PO Box 41882, Amsterdam, 1009 DB Netherlands.

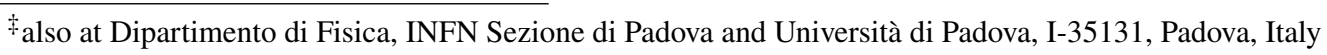


${ }^{16}$ University Mohammed V in Rabat, Faculty of Sciences, 4 av. Ibn Battouta, B.P. 1014, R.P. 10000 Rabat, Morocco.

${ }^{17}$ INFN, Sezione di Bologna, v.le C. Berti-Pichat, 6/2, Bologna, 40127 Italy.

${ }^{18}$ Università di Bologna, Dipartimento di Fisica e Astronomia, v.le C. Berti-Pichat, 6/2, Bologna, 40127 Italy.

${ }^{19}$ KVI-CART University of Groningen, Groningen, the Netherlands.

${ }^{20}$ INFN, Laboratori Nazionali del Sud, Via S. Sofia 62, Catania, 95123 Italy.

${ }^{21}$ North-West University, Centre for Space Research, Private Bag X6001, Potchefstroom, 2520 South Africa.

${ }^{22}$ Instituto Español de Oceanografía, Unidad Mixta IEO-UPV, C/ Paranimf, 1, Gandia, 46730 Spain.

${ }^{23}$ University Mohammed I, Faculty of Sciences, BV Mohammed VI, B.P. 717, R.P. 60000 Oujda, Morocco.

${ }^{24}$ Subatech, IMT Atlantique, IN2P3-CNRS, Université de Nantes, 4 rue Alfred Kastler - La Chantrerie, Nantes, BP 2072244307 France.

${ }^{25}$ Università di Salerno e INFN Gruppo Collegato di Salerno, Dipartimento di Matematica, Via Giovanni Paolo II 132, Fisciano, 84084 Italy.

${ }^{26}$ ISS, Atomistilor 409, Măgurele, RO-077125 Romania.

${ }^{27}$ University of Amsterdam, Institute of Physics/IHEF, PO Box 94216, Amsterdam, 1090 GE Netherlands.

${ }^{28}$ TNO, Technical Sciences, PO Box 155, Delft, 2600 AD Netherlands.

${ }^{29}$ Università degli Studi della Campania "Luigi Vanvitelli", Dipartimento di Matematica e Fisica, viale Lincoln 5, Caserta, 81100 Italy.

${ }^{30}$ Università La Sapienza, Dipartimento di Fisica, Piazzale Aldo Moro 2, Roma, 00185 Italy.

${ }^{31}$ Università di Bologna, Dipartimento di Ingegneria dell'Energia Elettrica e dell'Informazione "Guglielmo Marconi", Via dell'Università 50, Cesena, 47521 Italia.

${ }^{32}$ Cadi Ayyad University, Physics Department, Faculty of Science Semlalia, Av. My Abdellah, P.O.B. 2390, Marrakech, 40000 Morocco.

${ }^{33}$ University of the Witwatersrand, School of Physics, Private Bag 3, Johannesburg, Wits 2050 South Africa.

${ }^{34}$ Università di Catania, Dipartimento di Fisica e Astronomia "Ettore Majorana", Via Santa Sofia 64, Catania, 95123 Italy.

${ }^{35}$ INFN, LNF, Via Enrico Fermi, 40, Frascati, 00044 Italy.

${ }^{36}$ INFN, Sezione di Bari, via Orabona, 4, Bari, 70125 Italy.

${ }^{37}$ International Centre for Radio Astronomy Research, Curtin University, Bentley, WA 6102, Australia.

${ }^{38}$ University of Bari, Via Amendola 173, Bari, 70126 Italy.

${ }^{39}$ University Würzburg, Emil-Fischer-Straße 31, Würzburg, 97074 Germany.

${ }^{40}$ Western Sydney University, School of Computing, Engineering and Mathematics, Locked Bag 1797, Penrith, NSW 2751 Australia.

${ }^{41}$ Università di Salerno e INFN Gruppo Collegato di Salerno, Dipartimento di Fisica, Via Giovanni Paolo II 132, Fisciano, 84084 Italy.

${ }^{42}$ Università di Genova, Via Dodecaneso 33, Genova, 16146 Italy.

${ }^{43}$ Normandie Univ, ENSICAEN, UNICAEN, CNRS/IN2P3, LPC Caen, LPCCAEN, 6 boulevard Maréchal Juin, Caen, 14050 France.

${ }^{44}$ University of Granada, Dpto. de Física Teórica y del Cosmos \& C.A.F.P.E., 18071 Granada, Spain.

${ }^{45} \mathrm{NIOZ}$ (Royal Netherlands Institute for Sea Research), PO Box 59, Den Burg, Texel, 1790 AB, the Netherlands.

${ }^{46}$ Tbilisi State University, Department of Physics, 3, Chavchavadze Ave., Tbilisi, 0179 Georgia.

${ }^{47}$ Leiden University, Leiden Institute of Physics, PO Box 9504, Leiden, 2300 RA Netherlands.

${ }^{48}$ National Centre for Nuclear Research, 02-093 Warsaw, Poland.

${ }^{49}$ Institut Universitaire de France, 1 rue Descartes, Paris, 75005 France.

${ }^{50}$ University of Johannesburg, Department Physics, PO Box 524, Auckland Park, 2006 South Africa.

${ }^{51}$ Eberhard Karls Universität Tübingen, Institut für Astronomie und Astrophysik, Sand 1, Tübingen, 72076 Germany.

${ }^{52}$ Università di Pisa, Dipartimento di Fisica, Largo Bruno Pontecorvo 3, Pisa, 56127 Italy.

${ }^{53}$ Laboratoire Univers et Particules de Montpellier, Place Eugène Bataillon - CC 72, Montpellier Cédex 05, 34095 France.

${ }^{54}$ Friedrich-Alexander-Universität Erlangen-Nürnberg, Remeis Sternwarte, Sternwartstraße 7, 96049 Bamberg, Germany.

${ }^{55}$ Université de Haute Alsace, 68100 Mulhouse Cedex, France.

${ }^{56}$ University of Münster, Institut für Kernphysik, Wilhelm-Klemm-Str. 9, Münster, 48149 Germany.

${ }^{57}$ Utrecht University, Department of Physics and Astronomy, PO Box 80000, Utrecht, 3508 TA Netherlands.

${ }^{58}$ INFN, CNAF, v.le C. Berti-Pichat, 6/2, Bologna, 40127 Italy.

\section{Members of the JUNO Collaboration}

S. Ahmad ${ }^{10}$, J. P. A. M. de André ${ }^{4}$, E. Baussan ${ }^{4}$, C. Bordereau ${ }^{13,9}$, A. Cabrera ${ }^{2}$, C. Cerna ${ }^{13}$, G. Donchenko ${ }^{8}$, E. A. Doroshkevich ${ }^{5}$, M. Dracos ${ }^{4}$, F. Druillole ${ }^{13}$, C. Jollet ${ }^{13}$, L. N. Kalousis ${ }^{4}$, P. Kampmann ${ }^{1}$, K. Kouzakov ${ }^{8}$, A. Lokhov ${ }^{5,8}$, B. K. Lubsandorzhiev ${ }^{5}$, S. B. Lubsandorzhiev ${ }^{5}$, A. Meregaglia ${ }^{13}$, L. Miramonti ${ }^{3}$, F. Perrot ${ }^{13}$, L. F. Piñeres Rico ${ }^{4}$, A. Popov ${ }^{8}$, R. Rasheed ${ }^{13}$, M. Settimo ${ }^{11}$, K. Stankevich $^{8}$, H. Steiger ${ }^{7,12}$, M. R. Stock ${ }^{12}$, A. Studenikin ${ }^{8}$, A. Triossi ${ }^{4}$, W. Trzaska ${ }^{14}$, M. Vialkov ${ }^{8}$, B. Wonsak ${ }^{6}$, J. Wurtz ${ }^{4}$, and F. Yermia ${ }^{11}$.

${ }^{1}$ Forschungszentrum Jülich GmbH, Nuclear Physics Institute IKP-2, Jülich, Germany.

2 IJCLab, Université Paris-Saclay, CNRS/IN2P3, 91405 Orsay, France.

${ }^{3}$ INFN Sezione di Milano and Dipartimento di Fisica dell Università di Milano, Milano, Italy.

${ }^{4}$ IPHC, Université de Strasbourg, CNRS/IN2P3, F-67037 Strasbourg, France.

${ }^{5}$ Institute for Nuclear Research of the Russian Academy of Sciences, Moscow, Russia.

${ }^{6}$ Institute of Experimental Physics, University of Hamburg, Hamburg, Germany.

${ }^{7}$ Institute of Physics, Johannes-Gutenberg Universität Mainz, Mainz, Germany. 
${ }^{8}$ Lomonosov Moscow State University, Moscow, Russia.
${ }_{9}^{9}$ National United University, Miao-Li.
${ }^{10}$ Pakistan Institute of Nuclear Science and Technology, Islamabad, Pakistan.
${ }^{11}$ SUBATECH, Université de Nantes, IMT Atlantique, CNRS-IN2P3, Nantes, France.
${ }^{12}$ Technische Universität München, München, Germany.
${ }^{13}$ Univ. Bordeaux, CNRS, CENBG, UMR 5797, F-33170 Gradignan, France.
${ }^{14}$ University of Jyvaskyla, Department of Physics, Jyvaskyla, Finland. 